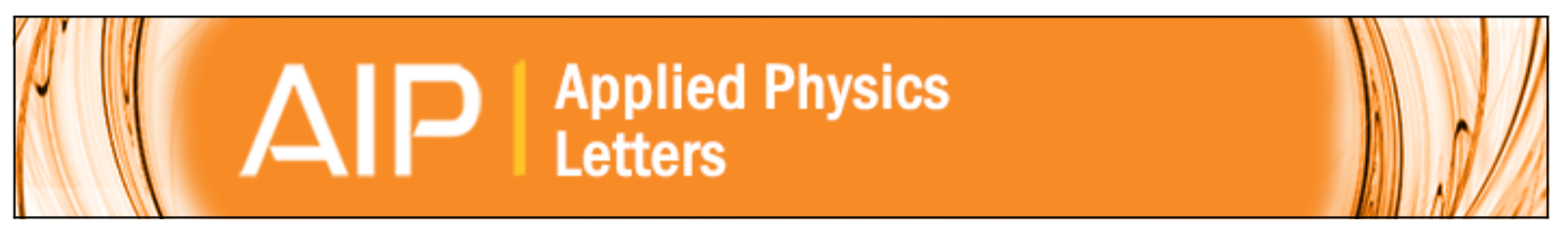

\title{
Probing bismuth ferrite nanoparticles by hard x-ray photoemission: Anomalous occurrence of metallic bismuth
}

Smita Chaturvedi, Indranil Sarkar, Mandar M. Shirolkar, U-Ser Jeng, Yi-Qi Yeh, Ranguwar Rajendra, Nirmalya Ballav, and Sulabha Kulkarni

Citation: Applied Physics Letters 105, 102910 (2014); doi: 10.1063/1.4895672

View online: http://dx.doi.org/10.1063/1.4895672

View Table of Contents: http://scitation.aip.org/content/aip/journal/apl/105/10?ver=pdfcov

Published by the AIP Publishing

\section{Articles you may be interested in}

Effect of europium substitution on the magnetic and optical properties of nanostructured bismuth ferrite AIP Conf. Proc. 1512, 1162 (2013); 10.1063/1.4791461

Structural, magnetic, and optical properties of $\mathrm{Pr}$ and $\mathrm{Zr}$ codoped $\mathrm{BiFeO} 3$ multiferroic ceramics J. Appl. Phys. 112, 094102 (2012); 10.1063/1.4761968

Correlation of spin and structure in doped bismuth ferrite nanoparticles

J. Appl. Phys. 111, $07 D 910$ (2012); 10.1063/1.3673814

Room temperature long range ferromagnetic ordering in (BiFeO3)1-x (PbTiO3)x nanocrystallites J. Appl. Phys. 109, 123911 (2011); 10.1063/1.3592281

Monitoring simultaneously the growth of nanoparticles and aggregates by in situ ultra-small-angle $\mathrm{x}$-ray scattering

J. Appl. Phys. 97, 054309 (2005); 10.1063/1.1855391

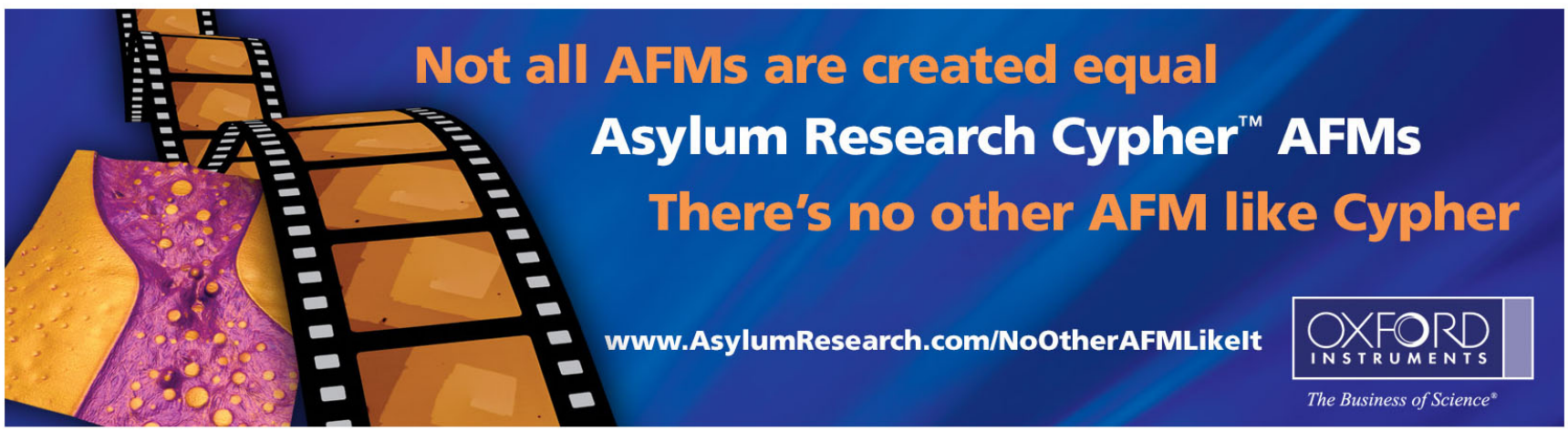




\title{
Probing bismuth ferrite nanoparticles by hard x-ray photoemission: Anomalous occurrence of metallic bismuth
}

\author{
Smita Chaturvedi, ${ }^{1}$ Indranil Sarkar, ${ }^{2}$ Mandar M. Shirolkar, ${ }^{3}$ U-Ser Jeng, ${ }^{4}$ Yi-Qi Yeh, ${ }^{4}$ \\ Ranguwar Rajendra, ${ }^{1}$ Nirmalya Ballav, ${ }^{1}$ and Sulabha Kulkarni ${ }^{1, a)}$ \\ ${ }^{1}$ Indian Institute of Science Education and Research, Dr. Homi Bhabha Road, Pune 411008, India \\ ${ }^{2}$ DESY Photon Science, Deutsches Elektronen-Synchrotron, 22607 Hamburg, Germany \\ ${ }^{3}$ Hefei National Laboratory for Physical Sciences at the Microscale, University of Science \\ and Technology of China, Hefei, Anhui 230026, People's Republic of China \\ ${ }^{4}$ National Synchrotron Radiation Research Center, 101, Hsin-Ann Road, Science Park, Hsinchu 3007-6, \\ Taiwan
}

(Received 10 April 2014; accepted 29 August 2014; published online 12 September 2014)

\begin{abstract}
We have investigated bismuth ferrite nanoparticles $(\sim 75 \mathrm{~nm}$ and $\sim 155 \mathrm{~nm})$ synthesized by a chemical method, using soft X-ray $(1253.6 \mathrm{eV})$ and hard X-ray $(3500,5500$, and $7500 \mathrm{eV})$ photoelectron spectroscopy. This provided an evidence for the variation of chemical state of bismuth in crystalline, phase pure nanoparticles. X-ray photoelectron spectroscopy analysis using $\mathrm{Mg} \mathrm{K} \alpha$ $(1253.6 \mathrm{eV})$ source showed that iron and bismuth were present in both $\mathrm{Fe}^{3+}$ and $\mathrm{Bi}^{3+}$ valence states as expected for bismuth ferrite. However, hard X-ray photoelectron spectroscopy analysis of the bismuth ferrite nanoparticles using variable photon energies unexpectedly showed the presence of $\mathrm{Bi}^{0}$ valence state below the surface region, indicating that bismuth ferrite nanoparticles are chemically inhomogeneous in the radial direction. Consistently, small-angle X-ray scattering reveals a core-shell structure for these radial inhomogeneous nanoparticles. C 2014 AIP Publishing LLC.

[http://dx.doi.org/10.1063/1.4895672]
\end{abstract}

Recently, there has been a growing interest in using nanostructured metal, semiconductor, and magnetic materials for variety of applications like biomedical, personal care, solar cells, fuel cells, electronic devices, etc. In investigating the properties of nanomaterials, size and shape are very crucial and widely investigated. However, internal chemical homogeneity within a nanoparticle (NP) is sparsely investigated, although it is a parameter of paramount interest that can render interesting functionalities to NPs. The lack of knowledge of the chemical homogeneity of NPs due to limited capabilities of conventional NP characterization tools, like X-ray Diffraction (XRD), Scanning Electron Microscopy (SEM), Transmission Electron Microscopy (TEM), Raman spectroscopy, X-ray Phoelectron Spectroscopy (XPS), etc., leads to the assumption that the nanoparticles are structurally and chemically homogeneous. However, few attempts made to understand the chemical state of nanoparticles at surfaces indeed showed that the nanoparticles can have surface segregation of one element over the other. ${ }^{1-4}$ In this manuscript, we employ a combination of photoemission spectroscopy, using soft X-rays from $\mathrm{Mg} \mathrm{K} \alpha(1253.6 \mathrm{eV})$ target and synchrotron radiation source with Hard X-ray Photoelectron Spectroscopy (HXPES), to probe chemical structure of a very important class of NP, namely, bismuth ferrite $\left(\mathrm{BiFeO}_{3}\right)$.

Bismuth ferrite (BFO) is a multiferroic material. A multiferroic material has more than one ferroic properties like ferroelectricity, ferromagnetism, and ferroelasticity, which are useful for many applications particularly in cases where a single material can perform multiple tasks. ${ }^{5-7}$ It crystallizes into a distorted perovskite with the rhombohedral $(R 3 c$ space group) structure but more symmetric tetragonal structure

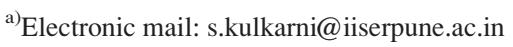

also has been reported. ${ }^{8}$ There is a great interest in BFO as it is the only known multiferroic with high transition temperatures, viz., ferroelectric transition $\mathrm{T}_{\mathrm{C}} \sim 1100 \mathrm{~K}$ and antiferromagnetic Néel temperature $\mathrm{T}_{\mathrm{N}} \sim 640 \mathrm{~K}$, both much above room temperature. ${ }^{6}$ The magnetic properties of $\mathrm{BFO}$ are attributed to $\mathrm{Fe}-\mathrm{O}$ bonds and ferroelectric properties arise due to Bi-O bonds in the distorted octahedra. This has led to extensive research primarily focused on physical property optimization, for example, magnetic property, in BFO by tailoring size of NPs. ${ }^{9-12}$ The work on NPs of BFO has shown that they have size dependent ferromagnetism. The appearance of ferromagnetism in BFO, which in the bulk form is basically a G-type antiferromagnetic material with canted spins on a spiral chain with period of $\sim 62 \mathrm{~nm}$ has been explained as due to abrupt termination of spiral, canted spin chain, as well as structural disorder due to the strain at surface. ${ }^{9}$ It has been speculated that BFO nanoparticles have a core-shell type of structure with an antiferromagnetic core and ferromagnetic shell. The appearance of ferromagnetism in BFO nanoparticles is evident from the appearance of coercivity which also has a size dependence, viz., coercivity keeps on increasing with decrease in the nanoparticle size. However, there is no detailed understanding about the thicknesses of cores and shells in different nanoparticles with variation of the size. It is very likely that these core-shell particles and their interfaces would be inhomogeneous but are not investigated in details. Thus, it is imperative to investigate the chemical homogeneity of BFO NPs that has been hitherto not well studied which may have profound effect on their observed properties.

There are many reports on BFO in which conventional soft XPS using $\mathrm{Al} \mathrm{K} \alpha$ or $\mathrm{Mg} \mathrm{K} \alpha \mathrm{X}$-ray sources has been used to determine the chemical state of $\mathrm{Bi}$ and Fe. ${ }^{13-15}$ In all such 
reports, $\mathrm{Bi}^{3+}$ and $\mathrm{Fe}^{3+}$ are observed in BFO both in bulk or nanostructures. However, so far there are no experiments in which chemical analysis of BFO nanoparticles has been made using both soft X-ray photoelectron spectroscopy and HXPES. HXPES using energies above $2 \mathrm{keV}$ has shown that the interfaces can be investigated non-destructively without ion bombardment by varying the photon energies. ${ }^{16-18}$ Ohtsuki et al. have shown in $\mathrm{Co}-\mathrm{TiO}_{2}$ thin films probed with soft X-ray and hard X-rays, possess different electronic structure in the surface region and bulk. ${ }^{19}$ They observe $\mathrm{Ti}^{4+}$ state in the surface region when probed with soft X-rays $(1200 \mathrm{eV})$ at a depth of $\sim 1 \mathrm{~nm}$ and metallic $\mathrm{Ti}^{3+}$ when probed with hard X-rays $(7940 \mathrm{eV})$ at a depth of $\sim 10 \mathrm{~nm}$. Thus, the chemical in-homogeneity existing at a deeper level was revealed. Here, we analyse chemical information as a function of depth, in BFO nanoparticles, by employing $\mathrm{X}$-rays of varying energy: $1253 \mathrm{eV}, 3500 \mathrm{eV}, 5500 \mathrm{eV}$, and $7500 \mathrm{eV}$. Our results clearly show that the BFO nanoparticles are radially inhomogeneous, revealing an interesting triple core shell structure, such that the intermediate shell region is composed of higher metallic Bi content, sandwiched between a low Bi content core and an oxidized outer shell. This result not only provides a very important information that has been generally neglected in modelling this very important class of multiferroics but also can be used to engineer the ferroelectric properties, as it has been theoretically predicted that metal-oxide interfaces can enhance ferroelectricity. ${ }^{20}$

The soft X-rays used here can give information from a depth of $\sim 1.2 \mathrm{~nm}$, while hard X-rays with photon energy $\sim 7500 \mathrm{eV}$ are able to yield information up to $\sim 10 \mathrm{~nm} .{ }^{16}$ The advantages of using HXPES in the analysis of materials are unique, as it provides non-destructive probe for depth dependent chemical structure analysis due to higher penetration of hard X-rays inside the solid surface compared to the soft $\mathrm{X}$-rays. This avoids unnecessary surface region alteration and provides the correct analysis of the valence state or chemical state of the constituting elements. ${ }^{17,18}$

BFO nanoparticles were synthesized using a soft chemical route combined with post synthesis annealing, reported earlier. $^{10,12}$ All the chemicals used are of analytical grade (99.9\% purity). Synthesis involves a reaction of $\mathrm{Bi}\left(\mathrm{NO}_{3}\right)_{3} \cdot 5 \mathrm{H}_{2} \mathrm{O}$ and $\mathrm{Fe}\left(\mathrm{NO}_{3}\right)_{3} \cdot 9 \mathrm{H}_{2} \mathrm{O}$ in the presence of tartaric acid at $353 \mathrm{~K}$, until the product is in sol-gel form. The sol-gel was heated in an oven at $423 \mathrm{~K}$. After washing in dilute nitric acid and ethanol, the obtained product was divided into two and one was annealed at $773 \mathrm{~K}$ and the other was annealed at $932 \mathrm{~K}$ for $2 \mathrm{~h}$ each. This gave two BFO powder samples BFO- 1 and BFO-2, respectively.

In order to know the morphology, purity, structure, and magnetic properties of the samples, we used conventional characterization tools: TEM, XRD, Raman spectroscopy, and magnetic measurements. TEM images of the samples were obtained using the FEI model Tecnai $200 \mathrm{G} 2$ with $\mathrm{LaB}_{6}$ electron source operating at $200 \mathrm{kV}$. The X-ray diffraction analysis of the samples was carried out using Bruker AXS D8 ADVANCE diffractometer. The data were collected for each sample in the slow scan mode from $15^{\circ}$ to $80^{\circ}$ in $10 \mathrm{~h}$. Raman analysis of the samples was performed at room temperature using Horiba Scientific Lab-RAM HR with laser wavelength $532 \mathrm{~nm}$. Magnetic measurements of the samples were performed on Physical Property Measurement System from Quantum Design (PPMS-VSM).

The XPS measurements were carried out at the HXPES facility at the P09 beamline at PETRA III (DESY, Hamburg, Germany) synchrotron source. The photon energy was varied between $3.5 \mathrm{keV}$ and $7.5 \mathrm{keV}$ using a $\mathrm{Si}(111)$ double crystal monochromator with an estimated energy resolution varying between $400 \mathrm{meV}$ and $800 \mathrm{meV}$ for low energy to high energy excitation. The data were recorded at normal photoemission geometry with photon incidence angle at $5^{\circ}$ to the surface of sample. The beam line is also equipped with fixed energy X-ray tube source. We used $\mathrm{Mg} \mathrm{K} \alpha(1253.6 \mathrm{eV})$ source in order to compare the soft X-ray data with HXPES data. The emitted photoelectrons were analysed using a hemispherical analyzer (SPECS Phoibos $225 \mathrm{HV}$ ). Small-angle x-ray scattering (SAXS) measurements were done using $15 \mathrm{keV}$ X-rays at BL23A SWAXS beamline in National Synchrotron Radiation Research Center, Taiwan. For this, BFO NPs were dispersed in ethanol.

The particle sizes were determined using TEM, Figs. 1(a) and 1(b). The particles are spherical in shape. The average particle size of sample BFO-1 is $75 \mathrm{~nm}$ and that of BFO2 is $155 \mathrm{~nm}$. The lattice parameters of the BFO nanoparticles are (see supplementary material ${ }^{21}$ ) consistent with the earlier reports. $^{22,23}$

The samples BFO-1 and BFO-2 were analysed for their structure and phase purity using XRD. Figs. 1(c) and 1(d) illustrate the Rietveld refinement of XRD data of BFO-1 and BFO2 , respectively. The detailed analysis showed (supplementary material $^{21}$ ) that the BFO samples BFO-1 and BFO-2 do not have any phase impurity as well as the nanoparticles of BFO are well crystallized, similar to thin films or single crystals in single rhombohedral phase $(R 3 c) .{ }^{6,24,25}$ However, this does not mean that samples are completely stoichiometric as expected. Lahmar et al. deliberately introduced in their thin film samples of BFO, $5 \%$ and $10 \%$ excess of Bi or Fe separately. ${ }^{25}$ They do not observe any structural variation up to $5 \%$ excess $\mathrm{Bi}$ or $\mathrm{Fe}$ in BFO thin films and rhombohedral $(R 3 c)$ phase was maintained. They also used Raman spectroscopy to find out if there are any changes in the vibration modes due to excess of $\mathrm{Bi}$ or $\mathrm{Fe}$. Interestingly, even the changes in Raman spectra appeared only after $10 \% \mathrm{Bi}$ or Fe addition.

We do not observe the structural transition in our BFO-1 and BFO-2 samples but the Raman spectra of both BFO-1 and BFO-2 samples as illustrated in Fig. 2 show some variations in the vibration modes in $70 \mathrm{~cm}^{-1}$ to $300 \mathrm{~cm}^{-1}$ and $425 \mathrm{~cm}^{-1}$ to $700 \mathrm{~cm}^{-1}$ regions. The wide range spectrum from $70 \mathrm{~cm}^{-1}$ to $700 \mathrm{~cm}^{-1}$ is given in supplementary material. ${ }^{21}$ But according to Chen et al., these changes can be attributed to the size dependence of nanoparticles. ${ }^{26}$ Therefore, from XRD and Raman analysis we conclude that the Bi or Fe related nonstoichiometry even if present (either overall or inhomogeneously) is less than $5 \%$ and difficult to detect by XRD and changes in the Raman spectra are due to size effect. Obviously XRD is not sensitive enough here to give us information about excess Bi or Fe less than 5\%. Thus, it is not possible with either XRD or Raman Spectroscopy to infer about any composition variation arising inside the nanoparticles of BFO here. The chemical homogeneity or inhomogeneity has important implications. ${ }^{20}$ From the point of view that core and 

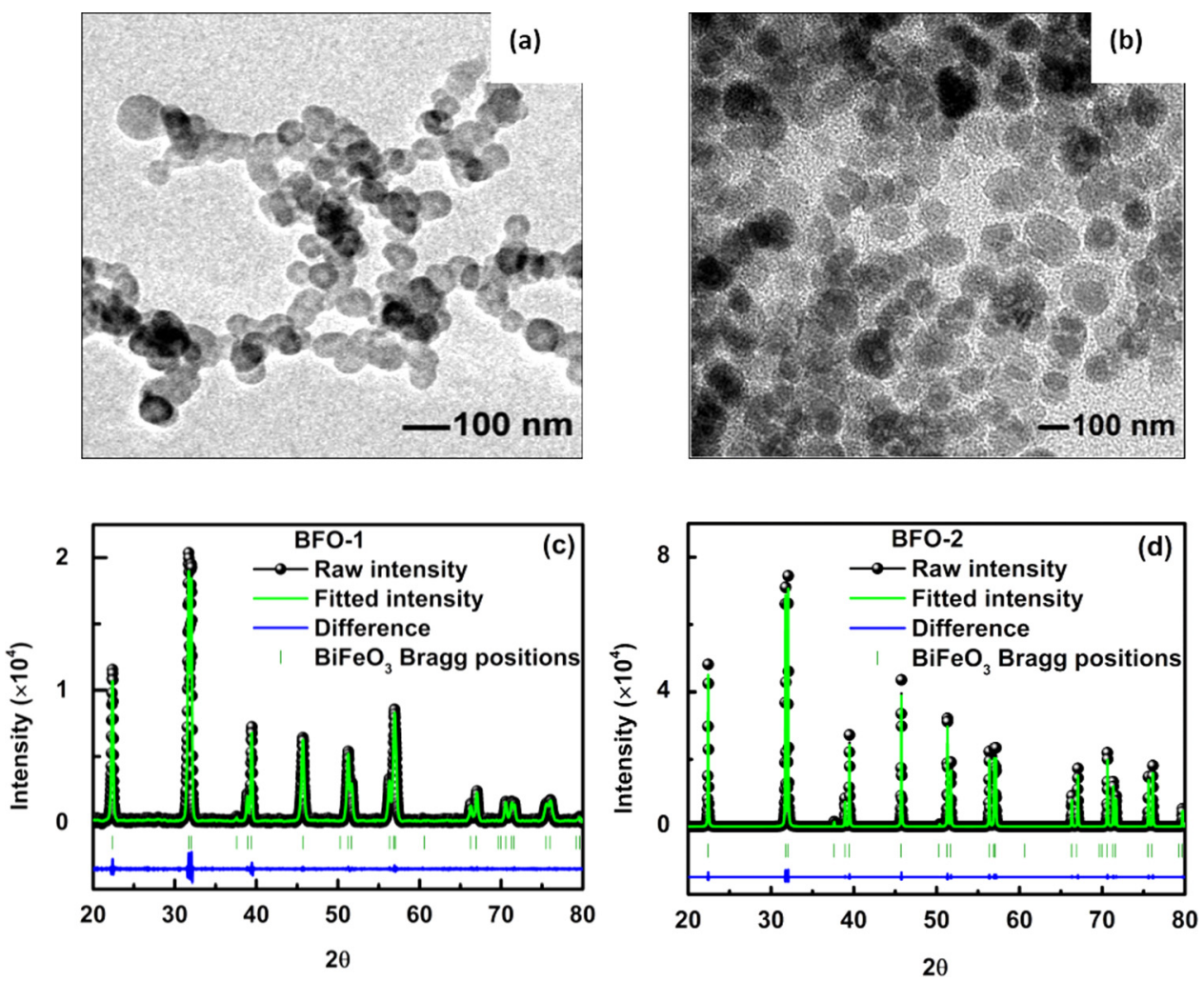

FIG. 1. TEM of BFO-1and BFO-2 ((a) and (b)) and Rietveld refined XRD pattern of BFO-1 and BFO-2 ((c) and (d)).

shell structure should have some interface (sharp or diffuse), its understanding using a non-destructive technique is important. However, the HXPES analysis is far from this simplistic analysis, as will be discussed below.

We do HXPES analysis of well characterized BFO samples BFO-1 and BFO-2. Survey scans of both the samples (see supplementary material ${ }^{21}$ ) show peaks only due to $\mathrm{Fe}, \mathrm{Bi}, \mathrm{O}$, and $\mathrm{C}$ and no impurities. With the well characterized core-shell BFO particles we proceed to the XPS analysis in order to investigate the issue of chemical homogeneity in the BFO samples.

The detailed HXPES scans of Fe $2 p(700-730 \mathrm{eV})$, Bi $4 \mathrm{f}$ $(154-170 \mathrm{eV})$, and $\mathrm{O} 1 \mathrm{~s}(525-535 \mathrm{eV})$ for both the BFO samples were made. Figs. 3(a) and 3(b) illustrate the HXPES for BFO-1 and BFO-2 samples in the Bi 4 f region (154-170 eV). Other spectra can be seen in supplementary material. ${ }^{21}$

The $\mathrm{Bi}$ 4f spectra were obtained with $1253.6 \mathrm{eV}$, $3500 \mathrm{eV}, 5500 \mathrm{eV}$, and $7500 \mathrm{eV} \mathrm{X}$-ray photon energies. In every spectrum, there are two doublets corresponding to $\mathrm{Bi}$ $4 \mathrm{f}_{7 / 2}$ and $\mathrm{Bi} 4 \mathrm{f}_{5 / 2}$. For sample BFO-1, the Bi peaks appear as follows: $\mathrm{Bi} 4 \mathrm{f}_{7 / 2}\left(\mathrm{Bi}^{3+}\right)$ at $158.8 \mathrm{eV}, \mathrm{Bi} 4 \mathrm{f}_{7 / 2}\left(\mathrm{Bi}^{0}\right)$ at $156.8 \mathrm{eV}$, $\mathrm{Bi} 4 \mathrm{f}_{5 / 2}\left(\mathrm{Bi}^{3+}\right)$ at $164.1 \mathrm{eV}$, and $\mathrm{Bi} 4 \mathrm{f}_{5 / 2}\left(\mathrm{Bi}^{0}\right)$ at $162.1 \mathrm{eV}$. For the $\mathrm{BFO}-2$ sample, the $\mathrm{Bi}$ peaks, $\mathrm{Bi} 4 \mathrm{f}_{7 / 2}\left(\mathrm{Bi}^{3+}\right)$ at $158.8 \mathrm{eV}$, $\mathrm{Bi} 4 \mathrm{f}_{7 / 2}\left(\mathrm{Bi}^{0}\right)$ at $156.5 \mathrm{eV}$, Bi $4 \mathrm{f}_{5 / 2}\left(\mathrm{Bi}^{3+}\right)$ at $164.1 \mathrm{eV}$, and $\mathrm{Bi}$ $4 \mathrm{f}_{5 / 2}\left(\mathrm{Bi}^{0}\right)$ at $161.8 \mathrm{eV}$ are very close to those observed in BFO-1 sample. The $\mathrm{Bi} 4 \mathrm{f}_{7 / 2}\left(\mathrm{Bi}^{3+}\right)$ and $\mathrm{Bi} 4 \mathrm{f}_{5 / 2}\left(\mathrm{Bi}^{3+}\right)$ positions are in good agreement with the data obtained using $\mathrm{Al} / \mathrm{Mg}$ laboratory XPS source. ${ }^{13-15}$ In Figs. 3(a) and 3(b), the spectra for BFO-1 and BFO-2 obtained using the $\mathrm{Mg} \mathrm{K} \alpha$ $(1253.6 \mathrm{eV})$ source are also shown. Here, we observe only two peaks corresponding to $\mathrm{Bi} 4 \mathrm{f}_{7 / 2}\left(\mathrm{Bi}^{3+}\right)$ and $\mathrm{Bi} 4 \mathrm{f}_{5 / 2}\left(\mathrm{Bi}^{3+}\right)$ at $158.8 \mathrm{eV}$ and $164.1 \mathrm{eV}$, respectively. The spin-orbit splitting is $5.3 \mathrm{eV}$ in both the samples. This is quite consistent with the earlier XPS analysis of BFO.

This observation of an additional peak in BFO-1 and BFO-2 recorded with HXPES shows that the nanoparticles of bismuth ferrite have $\mathrm{Bi}$ chemical states varying with depth. The additional peaks observed on the low binding energy side of $\mathrm{Bi}^{3+}$ peaks can be attributed to the zero
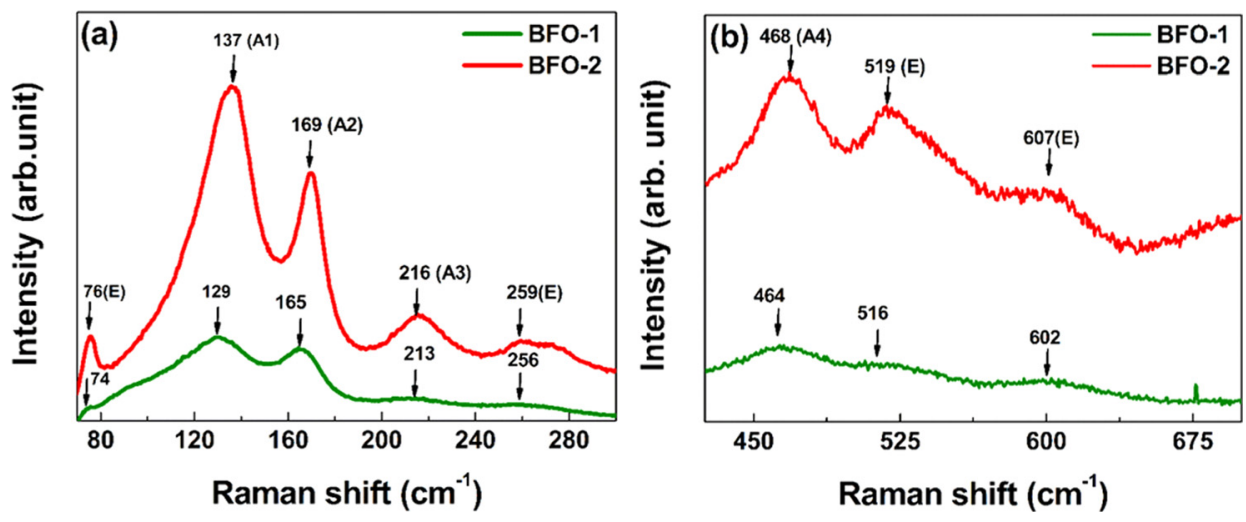

FIG. 2. Raman spectra of BFO-1 and BFO-2 showing shifting of modes due to increment in particle size in region $70-300 \mathrm{~cm}^{-1}$ (a) and $425-700 \mathrm{~cm}^{-1}$ (b). 

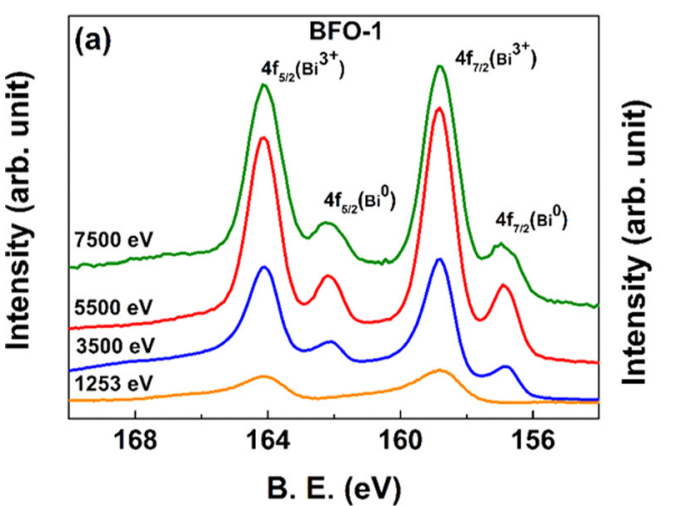

FIG. 3. Bi 4f spectra of BFO-1 (a) and BFO-2 (b) obtained using the Mg K $\alpha$ $(1253.6 \mathrm{eV})$ source along with HXPES. valence or metallic state of bismuth $\left(\mathrm{Bi}^{0}\right)$ in bismuth ferrite. Metallic peak is not observed in XPS obtained using Mg K $\alpha$ source. The signal due to $\mathrm{Mg} \mathrm{K} \alpha$ source emanates from surface to $\sim 1 \mathrm{~nm}$ depth. The peak observed at $158.8 \mathrm{eV}$ belongs to $\mathrm{Bi} 4 \mathrm{f}_{7 / 2}$ and that at $164.1 \mathrm{eV}$ belongs to $\mathrm{Bi} 4 \mathrm{f}_{5 / 2}$ in the $3^{+}$ states, respectively. The observed peaks are due to $\mathrm{Bi}^{3+}$ and $\mathrm{Fe}^{3+}$ states in $\mathrm{BFO}$ and peaks due to $\mathrm{Bi}_{2} \mathrm{O}_{3}$ or other oxides of $\mathrm{Fe}$ may get removed or not distinguished. Even if any oxides other than $\mathrm{BFO}$ were present, they were not detectable in XRD or XPS. In our case, we did not clean the samples with $\mathrm{Ar}$ ion bombardment. But here is an interesting observation of metallic $\mathrm{Bi}\left(\mathrm{Bi}^{0}\right)$ at $\sim 162 \mathrm{eV}\left(\mathrm{Bi} 4 \mathrm{f}_{5 / 2}\right)$ and $\sim 156.5 \mathrm{eV}\left(\mathrm{Bi}_{4 / 2}\right)$. It is evident from Figs. 3(a) and 3(b) that in both the cases the intensity of metallic $\mathrm{Bi}^{0}$ peak starts decreasing substantially at $7500 \mathrm{eV}$ photon energy. This would mean that the antiferromagnetic nanoparticles of BFO (with $\mathrm{Bi}^{3+}$ and $\mathrm{Fe}^{3+}$ ) are covered with a ferromagnetic shell in which metallic bismuth is present. In the depth of $\sim 1 \mathrm{~nm}$ from the surface, some metallic part may get oxidized and not detected in any technique used here. Thus, the BFO nanoparticles are graded in the radial direction with metallic component. It is interesting to find that appearance of this metallic component does not alter the physical structure of NPs, as revealed by XRD and Raman spectroscopy measurements. This could be related to the fact that, for low concentration of metallic $\mathrm{Bi}$ there is no observable structural change, has been also found in XRD and Raman spectroscopy studies of BFO thin films, having metallic Bi up to 5\% and $10 \%$, respectively, for the two structural characterization studies. $^{25}$

Such microscopic chemical analysis leading to the observation of metallic bismuth is extremely important in understanding and engineering the changes in the ferroelectricity in these materials. It has been shown theoretically that metal-oxide interfaces enhance the ferroelectricity. ${ }^{20}$ Thus, this finding of metallic bismuth in contact with oxide layers can be used to understand as well as tune the ferroelectric property in BFO. Furthermore, it has been reported ${ }^{25}$ that the ferroelectric leakage current improvement occurs in low thickness regime of BFO nanomaterials, which may be now related to the presence of this metallic component.

Fig. 4(a) shows a peak fitting analysis of HXPES spectra of BFO nanoparticles. The data have been fitted using UNIFIT, XPS fitting routine that employs a non-linear least squares curve-fitting algorithm of Marquardt and Levenberg, assuming a Shirley background. ${ }^{27}$ The data could be fitted with doublet structure corresponding to $4 \mathrm{f}_{7 / 2}$ and $4 \mathrm{f}_{5 / 2}$ core level of metallic $\mathrm{Bi}$ and $\mathrm{Bi}^{3+}$. Clearly the peak area ratio of 4f $\mathrm{Bi}^{0} / 4 \mathrm{f} \mathrm{Bi}^{3+}$ (Fig. 4(b)) shows a non-monotonic behavior as function of excitation photon energy. The ratio increases from 0 at $1.25 \mathrm{keV}$ to 0.27 for excitation energy of $3.5 \mathrm{keV}$. However, as the photon energy is increased above $3.5 \mathrm{keV}$, the ratio decreases to 0.06 at $7.5 \mathrm{keV}$. The corresponding inelastic mean free path for $\mathrm{Bi}_{2} \mathrm{O}_{3}$ based on Tanuma et al. (TPP-2 M) for $1.25 \mathrm{keV}, 3.5 \mathrm{keV}, 5.5 \mathrm{keV}$, and $7.5 \mathrm{keV}$ excitation energy are $\sim 2 \mathrm{~nm}, \sim 4.8 \mathrm{~nm}, \sim 7 \mathrm{~nm}$, and $\sim 9.1 \mathrm{~nm}$, respectively. ${ }^{28}$ This clearly indicates that we have metallic $\mathrm{Bi}$ rich region at about $4-5 \mathrm{~nm}$ depth in the $\mathrm{BFO}$ nanoparticles.

To verify the existence of the core-shell-shell structure of BFO NPs, SAXS measurements were carried out using 15 $\mathrm{keV} \mathrm{X}$-rays at BL23A SWAXS beamline in National Synchrotron Radiation Research Center, Taiwan. ${ }^{29}$ Fig. 5 shows the SAXS spectrum of BFO-2 NPs. The data have
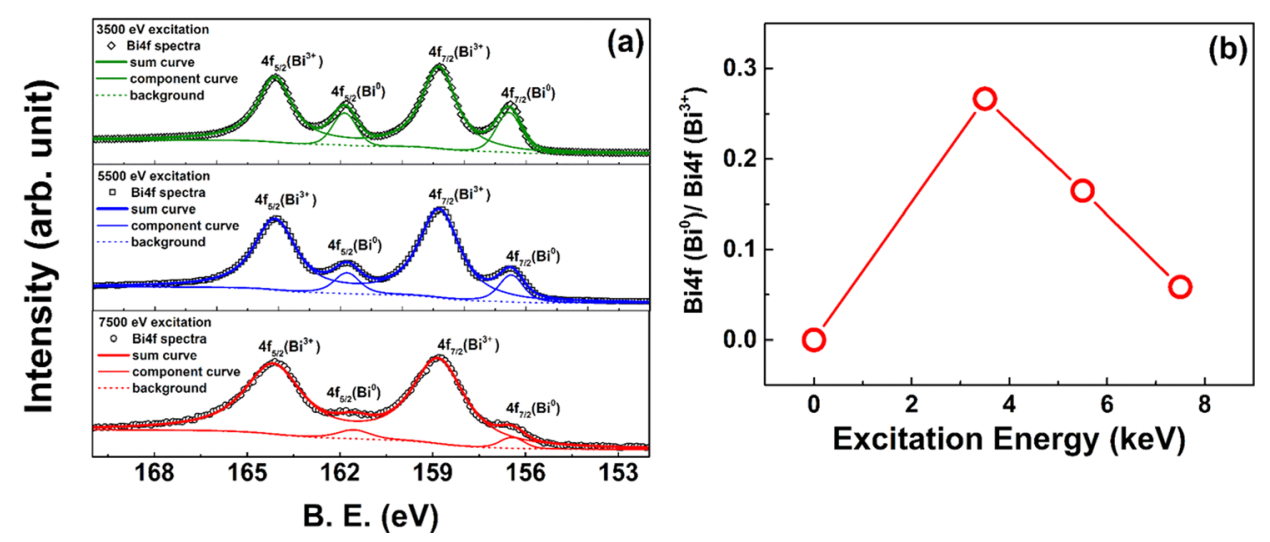

FIG. 4. Peak fitting analysis of HXPES spectra for $\mathrm{Bi} 4 \mathrm{f}$ region (a) and ratio of $\mathrm{Bi} 4 \mathrm{f}\left(\mathrm{Bi}^{0}\right)$ and $\mathrm{Bi} 4 \mathrm{f}\left(\mathrm{Bi}^{3+}\right)$ as a function of excitation energy (b) for sample BFO-2. 


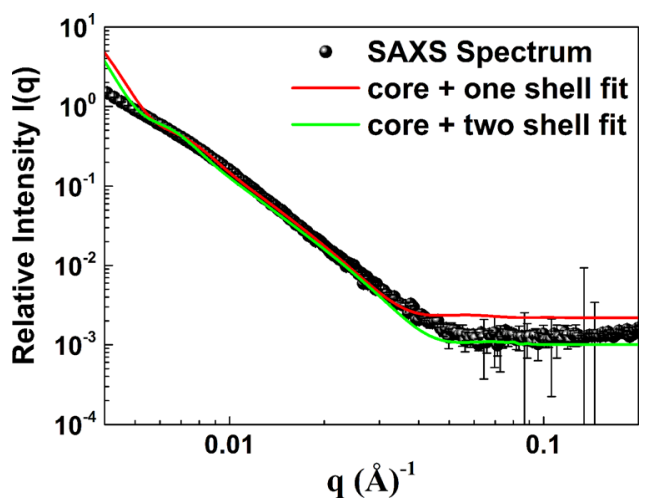

FIG. 5. Circle (O) shows the SAXS spectrum of BFO-2 NPs, having $\sim 155 \mathrm{~nm}$ diameter, with $25 \%$ polydispersity. Red line shows a model fitting with one core and one shell. Green line shows a model fitting with one core and two outer shells. The fitting has been carried out using nonlinear leastsquares fit under the IGOR platform. Here, the scattering wavevector $q=4 \pi \lambda^{-1} \sin \theta$ is defined by X-ray wavelength $\lambda$ and scattering angle $2 \theta$.

been fitted using a model of polydisperse core with $\mathrm{N}$ shells adopted from the Igor Macros. ${ }^{30}$

The SAXS spectrum has a much better fit (green line) with a core and two outer shells compared to a core and one outer shell (red-line). Hence, clearly the BFO NPs show a core-shell-shell structure as revealed by HXPES measurements as well. The obtained core diameter is $126 \mathrm{~nm}$, first shell and outermost shell thicknesses are $12.1 \mathrm{~nm}$ and $2 \mathrm{~nm}$, respectively. The corresponding fitted scattering length densities for core, first shell, and outermost second shell are $3.95 \times 10^{-5}, 6.21 \times 10^{-5}$, and $1 \times 10^{-5} \AA^{-2}$, respectively. Clearly the outermost shell has lowest electron density, while the intermediate shell has highest electron density. This could be explained by the fact that from HXPES measurements we find that the outer shell has bismuth oxide in combination with $\mathrm{BFO}$, while the intermediate shell has metallic $\mathrm{Bi}$ in addition to $\mathrm{BFO}$ which would render this layer a correspondingly higher electron density.

To conclude, bismuth ferrite nanoparticles of two sizes, viz., $75 \mathrm{~nm}$ and $155 \mathrm{~nm}$ were synthesized using a chemical method. The samples did not have any contamination and were phase pure. The XPS analysis using soft X-rays $(1.25 \mathrm{keV})$ showed $\mathrm{Bi}^{3+}$ valence state in the surface region. However, the HXPES analysis between $3.5 \mathrm{keV}$ and $7.5 \mathrm{keV}$ showed an additional valence for $\mathrm{Bi}$, viz., $\mathrm{Bi}^{0}$ or metallic state. Based on these results, the BFO nanoparticles can be modelled as chemically graded system with core-shell structure that have both $\mathrm{Bi}^{0}$ and $\mathrm{Bi}^{3+}$ valence states, such that a region of intermediate shell rich in metallic bismuth is formed, encapsulated between bismuth oxide rich core and an outer shell. The core-shell structure model is verified using SAXS measurement. This observation provides an extremely useful tool both for understanding and tuning the ferroelectric property in this very important class of materials.

The photoelectron spectroscopy work at PETRA-III beamline in DESY, Hamburg, Germany was carried out under the India-DESY collaborative Project No. 20110707 supported by DST, India. The authors thank DST and
DESY for the support. The authors thank DST, India Nano-Mission Initiative Project SR/NM/NS-42/2009. S.C. thanks DST, India (Grant no. SR/WOS/-A/PS50/2012(G)) and S.K. thanks UGC, India for the continuous support. Some preliminary measurements by Professor Krishnakumar Menon, SINP, India; magnetic measurement by Dr. Surjeet Singh, IISER, Pune, India; and discussions with Professor D. Pandey, BHU-IIT, India are gratefully acknowledged. The authors thank Neeraj Maheshwari for the technical help. The authors also thank the referee for the valuable suggestions which enabled to get more insight of the core-shell structure.

${ }^{1}$ U. Winkler, D. Eich, Z. H. Chen, R. Fink, S. K. Kulkarni, and E. Umbach, Chem. Phys. Lett. 306, 95 (1999).

${ }^{2}$ S. K. Kulkarni, U. Winkler, N. Deshmukh, P. H. Borse, R. Fink, and E. Umbach, Appl. Surf. Sci. 169-170, 438 (2001).

${ }^{3}$ L. Deng, W. Hu, H. Den, and S. Xiao, J. Phys. Chem. C 114, 11026 (2010).

${ }^{4}$ M. Asada, P. Gin, M. K. Endoh, S. K. Satija, and T. Koga, J. Phys.: Conf. Ser. 272, 012013 (2011).

${ }^{5}$ N. A. Hill and A. Filippetti, J. Magn. Magn. Mater. 242-245, 976 (2002).

${ }^{6}$ G. Catalan and J. F. Scott, Adv. Mater. 21, 2463 (2009).

${ }^{7}$ J. T. Heron, D. G. Schlom, and R. Ramesh, Appl. Phys. Rev. 1, 021303 (2014).

${ }^{8}$ Q. Y. He, H. Chu, J. T. Herron, S. Y. Yang, W. I. Liang, C. K. Kuo, H. J. Lin, P. Yu, C. W. Liang, R. J. Zeches et al., Nat. Commun. 2, 225 (2011).

${ }^{9}$ T. Park, G. C. Papaefthymiou, A. J. Viescas, A. R. Moodenbaugh, and S. S. Wong, Nano Lett. 7, 766 (2007).

${ }^{10}$ M. M. Shirolkar, R. Das, T. Maity, P. Poddar, and S. K. Kulkarni, J. Phys. Chem. C 116, 19503 (2012).

${ }^{11}$ F. Huang, Z. Wang, X. Lu, J. Zhang, K. Min, W. Lin, R. Ti, T. T. Xu, J. He, C. Yue, and J. Zhu, Sci. Rep. 3, 2907 (2013).

${ }^{12}$ S. Chaturvedi, M. M. Shirolkar, R. Rajendra, S. Singh, N. Ballav, and S. K. Kulkarni, J. Appl. Phys. 115, 123906 (2014).

${ }^{13}$ A. H. M. Gonzalez, A. Z. Simões, L. S. Cavalcante, E. Longo, J. A. Varela, and C. S. Riccardi, Appl. Phys. Lett. 90, 052906 (2007).

${ }^{14}$ R. Schafranek, J. D. Baniecki, M. Ishii, Y. Kotaka, and K. Kurihara, New J. Phys. 15, 053014 (2013).

${ }^{15}$ K. Jiang, J. J. Zhu, J. D. Wu, J. Sun, Z. G. Hu, and J. H. Chu, ACS Appl. Mater. Interfaces 3, 4844 (2011).

${ }^{16}$ C. S. Fadley, Nucl. Instrum. Methods Phys. Res., Sect. A 601, 8 (2009).

${ }^{17}$ J. J. Kim, E. Ikenaga, M. Kobata, A. Takeuchi, M. Awaji, H. Makino, P. P. Chen, A. Yamamoto, T. Matsuoka, D. Miwa, Y. Nishino, T. Yamamoto, T. Yao, and K. Kobayashi, Appl. Surf. Sci. 252, 5602 (2006).

${ }^{18}$ K. Kobayashi, Nucl. Instrum. Methods Phys. Res., Sect. A 601, 32 (2009).

${ }^{19}$ T. Ohtsuki, A. Chainani, R. Eguchi, M. Matsunami, Y. Takata, M. Taguchi, Y. Nishino, K. Tamasaku, M. Yabashi, T. Ishikawa, M. Oura, Y. Senba, H. Ohashi, and S. Shin, Phys. Rev. Lett. 106, 047602 (2011).

${ }^{20}$ M. Stengel, D. Wanderbilt, and N. A. Spladin, Nat. Mater. 8, 392 (2009).

${ }^{21}$ See supplementary material at http://dx.doi.org/10.1063/1.4895672 for bismuth ferrite nanoparticles.

${ }^{22}$ G. Biasotto, A. Z. Simões, C. R. Foschini, S. G. Antônio, M. A. Zaghete, and J. A. Varela, Process. Appl. Ceram. 5, 171-179 (2011).

${ }^{23}$ S. M. Selbach, T. Tybell, M. Einarsrud, and T. Grande, Chem. Mater. 19, 6478 (2007).

${ }^{24}$ X. Qi, M. Wei, Y. Lin, Q. Jia, D. Zhi, J. Dho, M. G. Blamire, and J. L. MacManus-Driscoll, Appl. Phys. Lett. 86, 071913-071915 (2005).

${ }^{25}$ A. Lahmar, K. Zhao, S. Habouti, M. Dietze, C.-H. Solterbeck, and M. EsSouni, Solid State Ionics 202, 1-5 (2011).

${ }^{26}$ P. Chen, X. Xu, C. Koenigsmann, A. C. Santulli, S. S. Wong, and J. L. Musfeldt, Nano Lett. 10, 4526-4532 (2010).

${ }^{27}$ R. Hesse, M. Weiß, R. Szargan, R. Streubel, and R. Denecke, J. Electron. Spectrosc. Relat. Phenom. 186, 44-53 (2013).

${ }^{28}$ S. Tanuma, C. J. Powell, and D. R. Penn, Surf. Interface Anal. 20, 77 (1993).

${ }^{29}$ U. Jeng, C.-H. Su, C.-J. Su, K.-F. Liao, W.-T. Chuang, Y.-H. Lai, J.-W. Chang, Y.-J. Chen, Y.-S. Huang, M.-T. Lee, K.-L. Yu, J.-M. Lin, D.-G. Liu, C.-F. Chang, C.-Y. Liu, C.-H. Chang, and K. S. Liang, J. Appl. Cryst. 43, 110-121 (2010).

${ }^{30}$ S. R. Kline, J Appl. Cryst. 39, 895 (2006). 